\title{
BMJ Open Impact of severe SARS-CoV-2 infection on nutritional status and subjective functional loss in a prospective cohort of COVID-19 survivors
}

To cite: Quilliot D, Gérard $\mathrm{M}$, Bonsack 0, et al. Impact of severe SARS-CoV-2 infection on nutritional status and subjective functional loss in a prospective cohort of COVID-19 survivors. BMJ Open 2021;11:e048948. doi:10.1136/ bmjopen-2021-048948

- Prepublication history for this paper is available online. To view these files, please visit the journal online (http://dx.doi org/10.1136/bmjopen-2021 048948).

Received 12 January 2021 Accepted 02 June 2021
Check for updates

(C) Author(s) (or their employer(s)) 2021. Re-use permitted under CC BY-NC. No commercial re-use. See rights and permissions. Published by BMJ.

For numbered affiliations see end of article.

Correspondence to Professor Didier Quilliot; d.quilliot@chru-nancy.fr

\section{ABSTRACT}

The nutritional sequelae of COVID-19 have not been explored in a large cohort study.

Objectives To identify factors associated with the change in nutritional status between discharge and 30 days postdischarge (D30). Secondary objectives were to determine the prevalence of subjective functional loss and severe disability at D30 and their associated factors.

Methods Collected data included symptoms, nutritional status, self-evaluation of food intake, Performance Status (PS) Scale, Asthenia Scale, self-evaluation of strength (SES) for arms and legs at discharge and at D30. An SES $<7$ was used to determine subjective functional loss. A composite criteria for severe disability was elaborated combining malnutrition, subjective functional loss and PS $>2$. Patients were classified into three groups according to change in nutritional status between discharge and D30 (persistent malnutrition, correction of malnutrition and the absence of malnutrition).

Results of 549 consecutive patients hospitalised for COVID-19 between 1 March and 29 April 2020, 130 died including 17 after discharge (23.7\%). At D30, 312 patients were at home, $288(92.3 \%)$ of whom were interviewed. Of the latter, $33.3 \%$ were malnourished at discharge and still malnourished at D30, while $23.2 \%$ were malnourished at discharge but no longer malnourished at D30. The highest predictive factors of persistent malnutrition were intensive care unit (ICU) stay (OR=3.42, 95\% Cl: 2.04 to 5.75), subjective functional loss at discharge $(\mathrm{OR}=3.26,95 \% \mathrm{Cl}: 1.75$ to 6.08$)$ and male sex $(\mathrm{OR}=2.39,95 \% \mathrm{Cl}: 1.44$ to 3.97$)$. Subjective functional loss at discharge $(76.8 \%)$ was the main predictive factor of subjective functional loss at D30 (26.3\%) (OR=32.6, 95\% Cl: 4.36 to 244.0$)$. Lastly, 8.3\% had a severe disability, with a higher risk in patients requiring an ICU stay (OR=3.39, 95\% $\mathrm{Cl}$ : 1.43 to 8.06$)$. Conclusion Patients who survived a severe form of COVID-19 had a high risk of persistent malnutrition, functional loss and severe disability at D30. We believe that nutritional support and rehabilitation should be strengthened, particularly for male patients who were admitted in ICU and had subjective functional loss at discharge.

Trial registration number NCT04451694.
Strengths and limitations of this study

- The nutritional sequelae of COVID-19 are unknown.

- Persons with diagnosed COVID-19 are also likely to exhibit decreased muscle function that cannot be readily assessed thus warranting a simple method to assess the evolution of muscle deficit.

- We used a self-evaluation of strength (SES) for arms and legs to detect the evolution in muscle strength which could be used to screen/follow the change in subjective functional loss of inpatients or outpatients given its simplicity.

- Identifying factors associated with the change in nutritional status between discharge and 30 days post-discharge (D30) and determining the prevalence of subjective functional loss and of serious disability at D30 as well as their associated factors should allow adapting the patient's care pathway after hospitalisation.

- The interest of the SES as a tool for sarcopenia screening should be evaluated and compared with an adequate reference test, that is, an objective value of muscle strength such as the hand grip.

\section{INTRODUCTION}

In March 2020, the east of France became one of the epicentres of COVID-19. While the clinical spectrum of SARS-CoV-2 infection appears to be wide, many patients were hospitalised with pneumonia. Few data have been published among patients with definite outcomes, including assessing the recovery period and possible nutritional and functional sequelae. ${ }^{1}$ Identifying patients at risk of malnutrition should allow adapting the patient's care pathway after hospitalisation.

Indeed, the multi-organ sequelae of COVID-19 beyond the acute phase of infection are increasingly being investigated as data and clinical experience in this timeframe accrue, with early reports suggesting residual effects of SARS-CoV-2 infection such 
as fatigue, dyspnoea, chest pain, cognitive disturbances, arthralgia and decline in quality of life, whereas malnutrition and muscle weakness, on the other hand, are poorly studied. $^{2}$

As with many acute infections, COVID-19 is a disease at high risk of malnutrition ${ }^{3}$ that can potentially mask muscle loss and/or loss of muscle function. The probability that the infected patient is already malnourished on admission to hospital is therefore high. Malnutrition is generally a factor of poor prognosis and should therefore be actively treated. Malnutrition is a subacute or chronic state in which a combination of negative energy balance and varying degrees of inflammatory activity leads to changes in body composition, diminished function and adverse outcomes. Moreover, inactivity and bed rest further accelerate muscle catabolism. Olfactory and gustatory dysfunction are common symptoms in patients with COVID- $19^{4}$ and may contribute to weight loss in other diseases, thereby increasing the risk of malnutrition. ${ }^{5}$ One study, conducted in Wuhan, China, showed that the prevalence of malnutrition based only on the Mini Nutritional Assessment in 182 elderly patients with COVID-19 was high (52.7\%), especially for those with diabetes mellitus, low calf circumference or low albumin. ${ }^{6}$

Persons with diagnosed COVID-19 are also likely to exhibit decreased muscle function resulting in secondary sarcopenia. Both myalgia and muscle loss have been described in COVID-19. ${ }^{7}$ Accordingly, COVID-19 is a situation where muscle mass cannot be readily assessed. Measuring muscle function, on the other hand, may be easier. Grip strength measurement comprises standardised protocols of measurement and features available robust and validated cut-off values, which is not the case for all lower limb muscle strength values. ${ }^{8}$ The use of grip strength is furthermore typically recommended as a supportive measure in the Global Leadership Initiative on Malnutrition (GLIM) ${ }^{910}$ and the second European Working Group on Sarcopenia in Older People consensus (EWGSOP2). ${ }^{10}$ During hospitalisation for COVID-19, handgrip strength is hence an appropriate supporting proxy under the cover of systematic decontamination, although time-consuming for healthcare teams. This test is however not easily usable to track the progress of muscle strength at home or in post-care facilities. The sarcopenic screening test is conversely a validated screening tool for remote consultation in primary care, ${ }^{11}$ although not adapted to hospitalised patients. ${ }^{12}$ A simpler method is thus necessary to assess the evolution of muscle deficit such as the evaluation of food intake by the self-evaluation of food intake (SEFI) using a visual analogue scale for diagnostic purposes, as well as to guide nutritional treatment. ${ }^{13}$ In a previous pilot study, ${ }^{14}$ we showed that a self-evaluation of strength (SES) for arms and legs enabled detecting progression in muscle strength measured with the hand grip test and could be used to screen/follow the change in subjective functional loss of inpatients or outpatients given its simplicity.
In keeping with the above, the objectives of this study were to identify factors associated with the change in nutritional status between discharge and 30 days post-discharge (D30) by distinguishing three groups of patients: one comprised of patients with persistent malnutrition at D30, one comprised of undernourished patients at discharge but no longer undernourished on D30 and one comprised of non-undernourished patients. Secondary objectives were to determine the prevalence of subjective functional loss and of serious disability at D30 as well as their associated factors.

\section{METHODS \\ Study design and participants}

This study, conducted as part of a prospective cohort study, included all adult inpatients ( $\geq 18$ years old) who were diagnosed with COVID-19 admitted in an intensive care unit (ICU) or non-ICU unit for COVID-19 patients at the Nancy Brabois University Hospital between 1 March 2020 and 29 April 2020 and subsequently discharged alive from hospital. The diagnosis of COVID-19 was based on a positive SARS-CoV-2 RT-PCR test on a nasopharyngeal sample and/or on a typical chest CT scan. ${ }^{15}$ The study was approved by the Research Commission of the University Hospital of Nancy and the requirement for informed consent was waived by the ethics commission.

To manage the flux of incoming patients, some were transferred to other hospitals after a few days. At discharge of the ICU or acute care unit, some patients were admitted to post-acute care facilities for ongoing skilled nursing care and rehabilitation.

The nutritional sequelae of COVID-19 are currently unknown. We therefore offered all hospital-discharged patients a phone consultation or teleconsultation D30 in order to assess their nutritional status and muscle function and to assess the degree of disability linked to the degree of malnutrition, subjective functional loss as well as limitation of activity, estimated by the WHO Performance Status (PS) score. We considered that the combination of these three criteria represents a severe disability for the patient and thus created a composite variable (disability criteria) based on these three parameters (malnutrition, subjective functional loss and PS $>2$ ).

\section{Demographics, comorbidities and hospitalisation data}

Patient characteristics and hospitalisation data were collected by manual review of electronic medical records.

Epidemiological, demographical, laboratory and outcome data were extracted from electronic medical records during hospitalisation.

Sociodemographic data included age $(<70 / \geq 70$ years old), sex, living alone, occupational activity (active vs unemployed and retired); smoking status (active or not) and daily alcohol consumption.

Health characteristics included comorbidities (hypertension, diabetes mellitus, cerebrovascular disease, cardiovascular disease, chronic lung disease) as well as 
the symptoms of COVID-19 (anosmia and dysgeusia, diarrhoea, dyspnoea, asthenia, food aversion).

Hospitalisation characteristics included: ICU admission (yes/no), time between symptom onset and hospitalisation (days) and length of stay (days).

\section{Symptoms}

Asthenia at discharge and at D30 was evaluated using a fatigue Visual Analogue Scale $(0-10) .{ }^{16}$ Dyspnoea at discharge and at D30 was evaluated by the French adaptation of the American Thoracic Society Scale, according to five levels (from 1 to 5 ). ${ }^{17}$

Health status at D30 was assessed using the WHO/ Zubrod PS Scale which categorises patients from 0 to $4 .^{18}$

\section{Nutritional assessment}

Nutritional status prior to hospitalisation, at admission, at discharge and at D30 were assessed using anthropometric measurements (body mass index (BMI)=body wt/ height $^{2}$ ) and weight loss (\%) compared with weight prior to illness. At D30, patients were instructed to use their own weighing scales. The following data were also recorded: Do you live: alone/in a couple/in an institution? Do you have difficulty shopping? Do you have help with shopping? Do you enrich your meals? Enrichment of the diet with fat? Food enrichment with proteins (milk protein, whey, etc)? Are you taking or have you taken oral nutritional supplements? Have you had enteral tube feeding? Have you had a consultation with a dietitian? Are you having trouble preparing meals? How many meals/ snacks per day? Do you have food tastes: which ones? How many times a day do you eat meat/fish/eggs? How many times a day do you eat dairy products/cheese? How many times a day do you eat fruit? Liquids: consumption/day $>1 \mathrm{~L} /$ day?

\section{SEFI and self-assessment of muscle function (SES) at discharge} and D30

Food intake was assessed using the 10-point verbal (AVeS) or visual (AViS) analogue scales (self-evaluation of food intake (SEFI)), ${ }^{19}$ from 0 to 10 . As suggested by Bouette $e t$ $a l,{ }^{19}$ a SEFI $<7$ was considered as the cut-off value.

SES was assessed at discharge and D30 using the 10-point verbal (AVeS) or visual (AViS) analogue scales via teleconsulting for evaluating arm and leg strength in comparison with patient strength prior to hospitalisation. In practice, patients were asked to evaluate their arm and leg strength in comparison with their strength prior to COVID-19. As suggested by Krznaric et al, ${ }^{14}$ patients were asked their degree of difficulty in lifting or carrying a weight, walking across the room, rising from a chair or bed and to evaluate these difficulties using a 10-point verbal or visual analogue scales $(10=$ same strength as before illness and $0=$ total loss of strength.

Assessment of physical activity at discharge and D30 were queried by completing the International Physical Activity Questionnaire-Short Form (IPAQ-SF) in order to estimate activity prior to COVID-19: activities were classified as low physical activity, moderate physical activity and high physical activity. ${ }^{20}$

The relevance of the SES to assess the change in muscle strength has previously been evaluated in a pilot study comparing hand grip changes. Changes in the handgrip test and SES recorded at admission and every 2 days during hospitalisation were analysed in a prospective cohort including inpatients until the required number of subjects was reached $(\mathrm{n}=26)$. At admission, handgrip strength was below $16 \mathrm{~kg}$ (for women) or $26 \mathrm{~kg}$ (for men) in 19/26 patients (73\%). During hospitalisation, handgrip values increased significantly $(26.5 \pm 14.0 \mathrm{~kg}$ vs $22.8 \pm 13.4, \mathrm{p}<0.0001)$ and SES $6.8 \pm 2.0$ vs $5.2 \pm 2.4$. Nineteen patients had a positive progression in handgrip and a positive self-evaluation of muscle strength whereas seven patients $(7 / 26)$ had no significant progression in handgrip values at the end of hospitalisation and five had no progression in SES values $\left(\chi^{2}=13.87 ; p<0.0001\right)$. Finally, SES enabled detecting $95 \%$ of patients with progression in muscle strength and detecting $71 \%$ patients without progression.

Malnutrition diagnosis was made according to the GLIM criteria and French recommendations (at least one phenotypical criterion and one etiological criterion). ${ }^{91}$ Severe malnutrition (SM) was defined following the French recommendations as weight loss $>10 \%$ than before COVID-19 or a BMI $<17 \mathrm{~kg} / \mathrm{m}^{2}(<18.5$ for patients $>70$ years old). MM was defined as weight loss $>5 \%$ than before COVID-19 or BMI $<18.5 \mathrm{~kg} / \mathrm{m}^{2}$ ( $<21$ for patients $>70$ years old). ${ }^{21} 22$

Explanatory variables:

1. Evolution of nutritional status was established by the change in nutritional status between discharge and D30: patients who were non-malnourished at discharge and at D30 (Discharge-/D30- group), patients malnourished at discharge but not malnourished at D30 (Discharge+/D30- group) and patients still malnourished at D30 (Discharge+/D30+ group).

2. Subjective functional loss at D30 assessed by SES.

3. Severe disability established by a composite indicator comprised of: malnutrition, subjective functional loss $($ SES $<7 / 10)$ and PS $>2$.

\section{Statistical analysis}

Continuous variables are expressed as mean $\pm \mathrm{SD}$ and categorical variables as absolute values and percentages. Paired Student's t-test, $\chi^{2}$, analysis of variance (ANOVA), Fisher's exact test and Wilcoxon tests were used to compare values of variables between groups, as appropriate. Pearson's $\chi^{2}$ test or Fisher's exact test was used to assess the association between each of the discrete variables and patients' malnutrition status. Variables significant at a 0.05 level were subsequently used in multivariate analyses.

A receiver operating characteristic (ROC) curve was used to assess the predictive performance of the SES compared with the reference test, namely the GLIM criteria for malnutrition diagnosis, after which the area under the ROC curve (AUC) was determined. Sensitivity, 


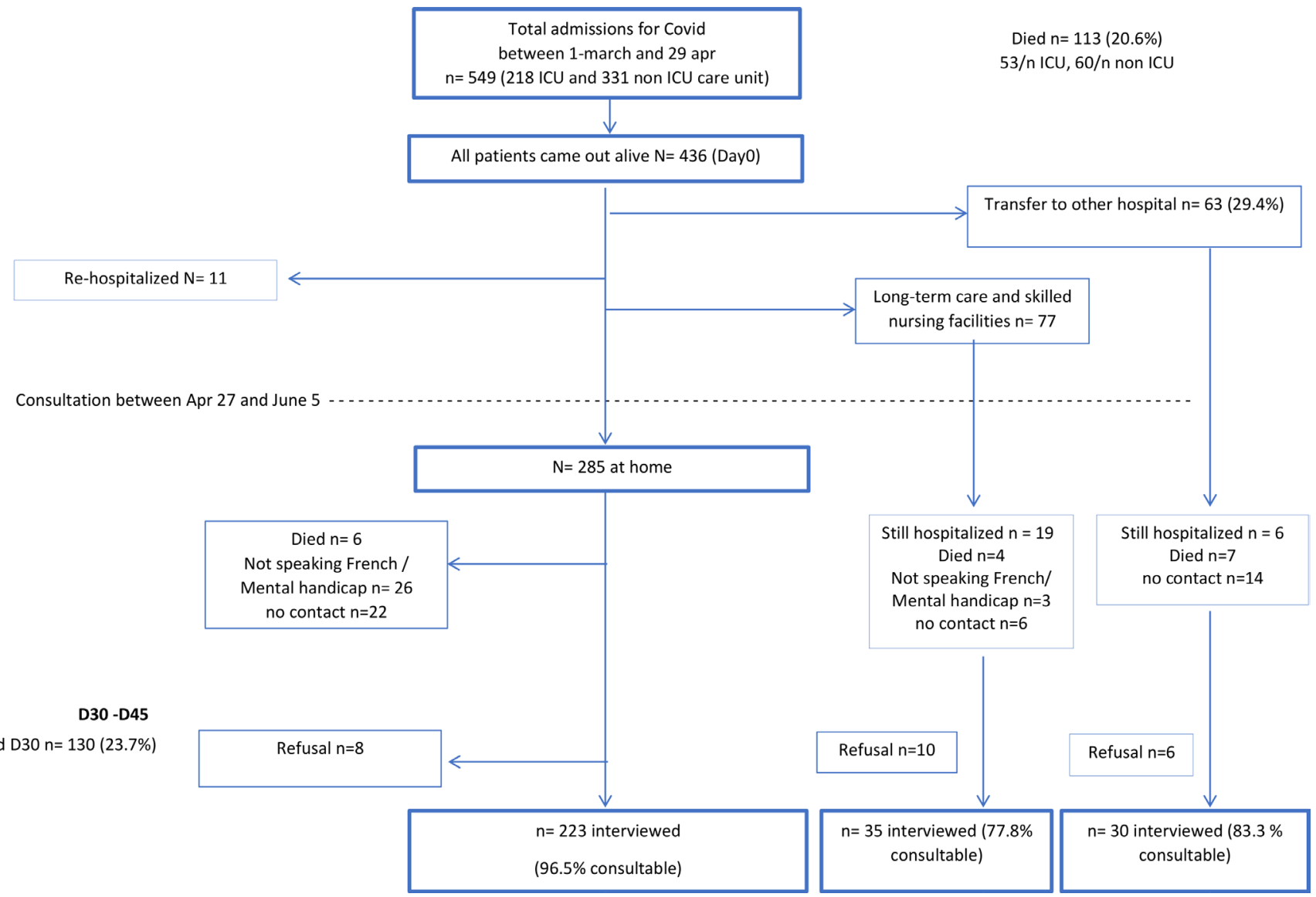

Figure 1 Flowchart of patients included in the study. ICU, intensive care unit.

specificity, positive and negative predictive values and their respective $95 \% \mathrm{CI}$, were calculated.

Factors associated with malnutrition, subjective functional loss and severe disability (composite indicator) were investigated by using a dichotomous logistic regression model with a stepwise selection method. Factors associated with the change in nutritional status were treated as dependent variables in the ordinal polychotomous logistic regression.

Data were recorded on Excel files. Statistical analysis was performed using Statistical Analysis Software V.9.4 (SAS Institute).

\section{RESULTS}

\section{Study cohort}

During the study period (from 1 March 2020 to 29 April 2020), 549 patients required admission to hospital due to severe illness, including $63 / 549(11.5 \%)$ who were discharged to another hospital in the first week due to lack of hospital beds (figure 1). Among the 549 patients with severe illness, $218(39.7 \%)$ required intensive care with or without mechanical ventilation, 53 of whom died $(24.3 \%)$. Three hundred and thirty-one patients $(60.3 \%)$ required non-ICU care and 60 died (18.1\%). Seventy-seven of the 436 surviving patients $(17.6 \%)$ were discharged to post-acute care facilities and 4 patients of this group died during the following month. Among the 296 patients at home, 11 were readmitted in acute care units during the month after discharge and 6 died. At day 30, 288 patients were interviewed. Twenty-four out of 383 patients refused the interview $(6.3 \%)$ whereas $29(7.6 \%)$ could not answer due to language barrier or learning disabilities. Forty-two (11.0\%) had given a wrong phone number or had not given their phone number or email address at admission.

\section{Population description}

Mean age of the 288 interviewed patients was $59.8 \pm 16.6$ years, $54.2 \%$ of whom were male. As described in table 1 , the majority had a low physical activity assessed by IPAQ-SF prior to contracting COVID-19 (56.3\%). Seventy-six per cent of these patients were overweight or obese. The most frequent comorbidities were cardiovascular diseases (hypertension, dyslipidaemia, diabetes mellitus and coronary artery disease). Anosmia was present in $42.7 \%$ of patients at admission and $11.8 \%$ at D30. Dyspnoea was a patient complaint in $22.6 \%$ at admission and rose to $77.7 \%$ at D30 (stage 1: $37.6 \%$; stage 2: $31.4 \%$; stage 3: $8.0 \%$, stage $4: 0.7 \%$ ). Cough was present in $25 \%$ at admission and in $6.3 \%$ at D30. Diarrhoea was present in $9.3 \%$ of patients at admission and $6.3 \%$ at D30.

Variations in anthropometric, muscle and disability parameters

BMI, weight (\%), SEFI and SES variations between admission, discharge and D30 are detailed in table 2. Mean initial 
Table 1 Patient characteristics at admission

\begin{tabular}{lc}
\hline Age (years) & $59.8 \pm 16.6$ \\
Sex (F/M) & $132 / 156(45.8 \% / 54.2 \%)$ \\
Living alone & $54(18.8 \%)$ \\
Couple & $132(45.8 \%)$ \\
Family & $96(33.3 \%)$
\end{tabular}

\begin{tabular}{|cc|}
$\begin{array}{l}\text { Retirement home } \\
\text { BMI class }\end{array}$ & $6(2.1 \%)$ \\
\hline $18.5-24.9$ & $69(24.3 \%)$ \\
$25-29.9$ & $112(39.4 \%)$ \\
$30-34.9$ & $67(23.6 \%)$ \\
$35-39.9$ & $24(8.5 \%)$ \\
$>40$ & $12(4.2 \%)$ \\
\hline
\end{tabular}

IPAQ (prior to COVID-19)

\begin{tabular}{|c|c|}
\hline Low & $162(56.3 \%)$ \\
\hline Moderate & $82(28.5 \%)$ \\
\hline High & $44(15.3 \%)$ \\
\hline \multicolumn{2}{|l|}{ Profession } \\
\hline Active & $117(41.7 \%)$ \\
\hline Health sector & $28(10.0 \%)$ \\
\hline Other professions & $89(31.7 \%)$ \\
\hline Inactive & $164(58.3 \%)$ \\
\hline Retired & $131(46.6 \%)$ \\
\hline Unemployed & $33(11.7 \%)$ \\
\hline Active smoking & $12(4.2 \%)$ \\
\hline Daily alcohol & $14(4.9 \%)$ \\
\hline ICU & $102(35.4 \%)$ \\
\hline \multicolumn{2}{|l|}{ Comorbidities } \\
\hline HBP & $113(39.2 \%)$ \\
\hline Coronary heart disease & $41(14.2 \%)$ \\
\hline Dyslipidaemia & $64(22.2 \%)$ \\
\hline Diabetes mellitus & $48(16.7 \%)$ \\
\hline Renal failure & $23(8.0 \%)$ \\
\hline Stroke & $9(3.1 \%)$ \\
\hline Asthma & $14(4.9 \%)$ \\
\hline Apnoea & $19(6.6 \%)$ \\
\hline Chronic obstructive bronchitis & $13(4.5 \%)$ \\
\hline Respiratory failure & $10(3.5 \%)$ \\
\hline Active cancer & $30(10.4 \%)$ \\
\hline Neurological disease & $13(4.5 \%)$ \\
\hline
\end{tabular}

BMI, body mass index; HBP, high blood pressure; ICU, intensive care unit; IPAQ, International Physical Activity Questionnaire.

BMI (BMI corresponding to stable weight before COVID19) was $29.1 \pm 5.9 \mathrm{~kg} / \mathrm{m}^{2}$ (mean weight $83.0 \pm 18.5 \mathrm{~kg}$ ), mean admission BMI was $28.5 \pm 6.1,27.2 \pm 5.7$ at discharge and $27.9 \pm 5.5$ at D30. Mean involuntary weight loss was $-2.7 \pm 4.2 \%$ before admission (delay $<1$ month), which persisted during hospitalisation $(-3.4 \pm 5.1 \%)$. On average, weight increased after discharge, up to D30 $(+2.8 \% \pm 3.6 \%$, $\mathrm{p}<0.0001)$. Lastly, 16 patients $(5.5 \%)$ continued their weight loss after discharge.

\section{Malnutrition prevalence}

As described in table 2, 20.7\% presented malnutrition at admission, with $6.6 \%$ exhibiting SM and $14.1 \%$ exhibiting MM. At discharge, $56.9 \%$ presented malnutrition: $27.2 \%$ exhibited SM and 29.7\% MM. At D30, 33.3\% presented persistent malnutrition: $13.2 \%$ with SM and $20.1 \%$ with MM $(\mathrm{p}<0.0001)$.

\section{Change in nutritional status}

One hundred and twenty-five patients $(43.4 \%)$ presented no malnutrition at discharge or at D30 (M-/M-), 67 patients (23.2\%) were malnourished at discharge but no longer malnourished at D30 (M+/M-) while $96(33.3 \%)$ were malnourished at discharge and still malnourished at D30 $(\mathrm{M}+/ \mathrm{M}+)$. Sixty-one percent of malnourished patients at discharge remained malnourished at D30. In this group, 16 patients $(5.5 \%)$ continued their weight loss after discharge.

As reported in table 3 , sex (male vs female, $\mathrm{p}<0.0011$ ), dyslipidaemia $(p=0.0301)$, immunotherapy $(p=0.0011)$, ICU admission $(\mathrm{p}<0.0001)$, post-acute care facilities (post-care facilities vs at home return, $\mathrm{p}<0.0001$ ), subjective functional loss at discharge $(\mathrm{p}<0.0011)$ and hospital duration ( $>7$ days vs $\leq 7$ days, $\mathrm{p}=0.0034$ ) were associated with unfavourable evolution of nutritional status.

Polychotomous stepwise logistic regression analysis revealed that the three strongest and most consistent predictors of change in nutritional status were: being admitted in ICU (OR=3.42; 95\% CI: 2.04 to 5.75), subjective functional loss at discharge ( $\mathrm{OR}=3.26 ; 95 \%$ CI: 1.75 to 6.08 ) and male sex (OR=2.39; $95 \%$ CI: 1.44 to 3.97$)$.

\section{Subjective functional loss}

Accuracy of the 10-point SES visual analogue scale score

The area under the ROC curve evaluating the performance of the SES visual analogue scale score for the diagnosis of malnutrition was 0.66 (95\% CI: 0.590 to $0.727)$. A threshold value of the SES visual analogue scale score equal to 7 allowed the best combination between sensitivity and Youden's index. For this threshold, the sensitivity for the diagnosis of malnutrition was $87.4 \%$, specificity was $33.0 \%$, the positive predictive value was $64.2 \%$ and the negative predictive value was $66.1 \%$.

An SES $<7 / 10$ (subjective functional loss) was observed in $76.8 \%$ of patients (mean $=5.0 \pm 2.6$ for arm and $4.9 \pm 2.6$ for legs). At D30, subjective functional loss prevalence decreased significantly: $26 \%$ had subjective functional loss $(\mathrm{p}<0.0001)$ with mean $8.1 \pm 2.0$ for arm and 8.0 2.0 for legs; $\mathrm{p}<0.0001$ (table 2). At D30, 26.2\% had subjective functional loss defined by SES $<7 / 10$ (mean $8.1 \pm 2.0$ for arm and 8.1 \pm 2.0 for legs; $\mathrm{p}<0.0001$ )

Bivariate and multivariate analysis (table 4) showed that D30- subjective functional loss was significantly 
Table 2 Change in nutritional status and muscle strength between admission and (post-)discharge $(n=288)$

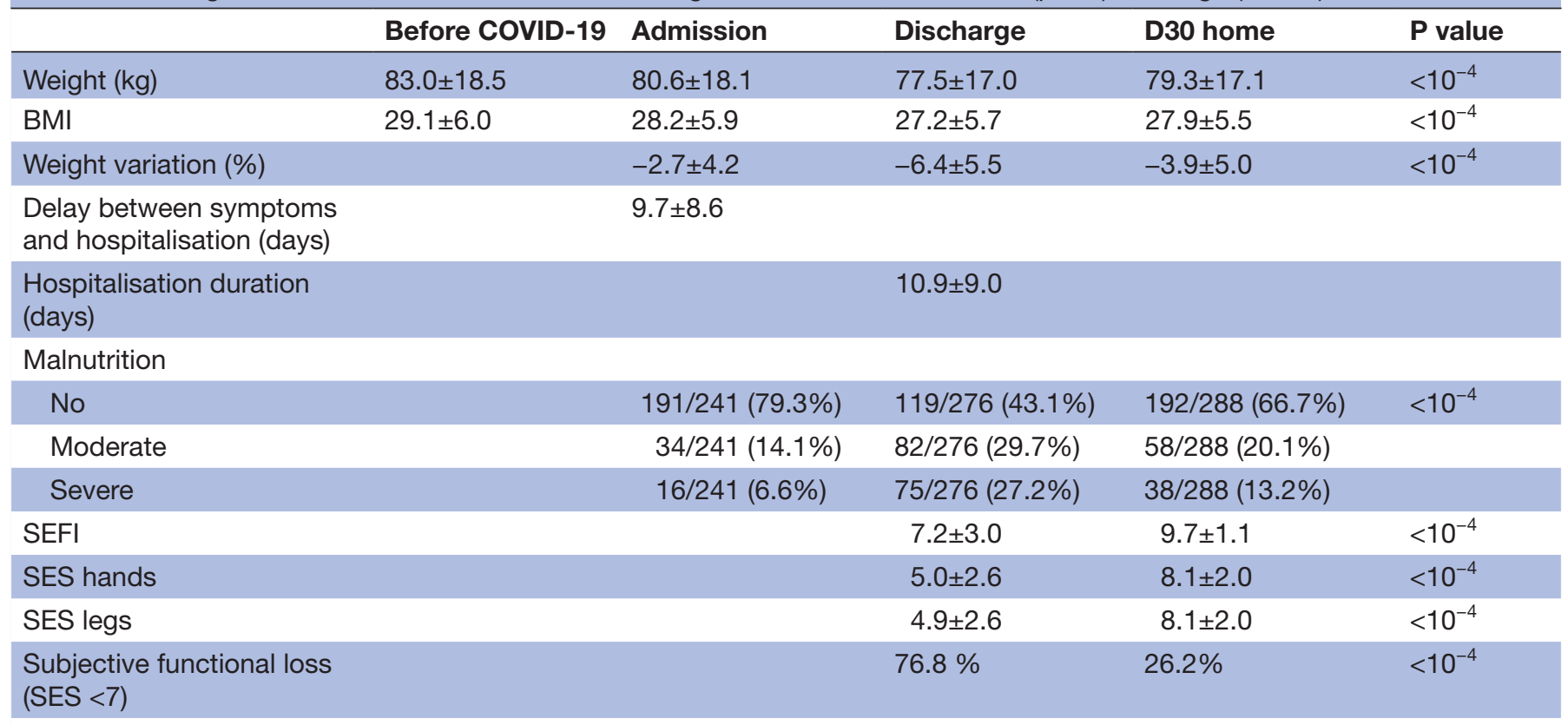

\section{Performance status}

\begin{tabular}{ll}
0 & $139 / 283(49.1 \%)$ \\
1 & $81 / 283(28.6 \%)$ \\
2 & $39 / 283(13.8 \%)$ \\
$3-4$ & $24 / 283(8.5 \%)$ \\
\hline
\end{tabular}

$\mathrm{BMI}$, body mass index (weight/T²); SEFI, self-evaluation of food intake; SES, self-evaluation of strength.

associated with stay in post-acute care facilities (vs at home) $(p=0.0089)$, ICU admission $(p=0.0239)$ and MM or SM $(p=0.0151)$, but not significantly with anosmia $(\mathrm{p}=0.0567)$ (table 4). In multivariate analysis (stepwise logistic regression), subjective functional loss at discharge was the main predictor of subjective functional loss at D30 (significant $\mathrm{OR}=32.6 ; 95 \% \mathrm{CI}: 4.36$ to 244.0 ) and post-acute care facilities (significant $\mathrm{OR}=2.79 ; 95 \% \mathrm{CI}$ : 1.42 to 5.48 )

\section{Composite disability criteria at D30}

Twenty-four patients $(8.5 \%)$ presented composite disability criteria combining a WHO PS $>2$, malnutrition and subjective functional loss (table 2). Eleven patients had SM and 13 had MM. In univariate analyses (table 5), disability criteria were significantly associated with sex (male) $(\mathrm{p}=0.0386)$ and ICU stay $(\mathrm{p}=0.0057)$ while negatively associated with anosmia $(\mathrm{p}=0.0302)$. In multivariate analysis (logistic regression), a stay in ICU was the main parameter associated with the disability criteria at D30 (OR=3.39; 95\% CI: 1.43 to 8.06$)$.

Of these 24 patients, 5 were admitted in post-care facilities. Nine were managed at home and required a physiotherapist, six pursued nutritional supplementations as well as protein supplementation after physical exercise and practiced exercises by themselves after advice. Six patients received dietary advice but refused physiotherapy and nutritional supplementation. Two patients did not wish to gain weight. Three patients had mostly respiratory issues and had oxygen at home, including one patient with lung ablation.

An additional four patients had serious difficulties to eat $($ SEFI $<7)$ due to anosmia $(n=2)$ or loss of appetite $(n=3)$ and 13 had severe fatigue $(\leq 5 / 10$ on the standard scale).

\section{DISCUSSION}

This is the first report on a prospective observational cohort study of COVID-19 specifically exploring nutritional status, subjective functional loss and disability at D30. The present study showed that, at D30, 33.3\% of these patients presented persistent malnutrition, with a higher risk in patients who had been admitted in ICU, had subjective functional loss at discharge and were of male sex. Furthermore, the novel use of a self-report of muscle strength enabled assessing the perception of loss of muscle function in everyday life after an infectious aggression. With this tool, prevalence of subjective functional loss at discharge was considerable and remained very high at D30 (26.3\%) and represented the main predictive factor of subjective functional loss at D30. Finally, at least $8.3 \%$ of patients had a severe disability which urgently necessitated home care assistance, and/ 
Table 3 Bivariate and multivariate analysis of change in nutritional status (OR from ordinal polychotomous stepwise logistic regression)

\begin{tabular}{|c|c|c|c|c|c|c|c|c|c|c|c|}
\hline & \multirow[b]{3}{*}{$\mathbf{N}$} & \multicolumn{2}{|c|}{$\begin{array}{l}\text { Change in } \\
\text { nutritional } \\
\text { status }\end{array}$} & \multicolumn{3}{|c|}{ Bivariate regression } & \multirow[b]{3}{*}{$P$ value } & \multicolumn{4}{|c|}{ Multivariate regression* } \\
\hline & & \multirow[b]{2}{*}{$\mathbf{n}$} & \multirow[b]{2}{*}{$\%$} & \multirow[b]{2}{*}{ OR } & \multicolumn{2}{|c|}{$95 \% \mathrm{Cl}$} & & \multirow[b]{2}{*}{ OR } & \multicolumn{2}{|c|}{$95 \% \mathrm{Cl}$} & \multirow[b]{2}{*}{$P$ value } \\
\hline & & & & & Upper & Lower & & & Upper & Lower & \\
\hline Age (years) & & & & & & & 0.6372 & & & & \\
\hline$<70$ & 208 & 65 & 33 & 1 & & & & & & & \\
\hline$>70$ & 80 & 30 & 38 & 1.12 & 0.69 & 1.82 & & & & & \\
\hline Sex & & & & & & & $<0.0001$ & & & & 0.0007 \\
\hline Male & 152 & 65 & 42.8 & 2.74 & 1.74 & 4.32 & & 2.39 & 1.44 & 3.97 & \\
\hline Female & 124 & 30 & 24.2 & 1 & & & & 1 & & & \\
\hline Dyslipidaemia & & & & & & & 0.0301 & & & & \\
\hline No & 212 & 66 & 31.1 & 1 & & & & & & & \\
\hline Yes & 64 & 29 & 45.3 & 1.78 & 1.06 & 3 & & & & & \\
\hline Immunotherapy & & & & & & & 0.0011 & & & & \\
\hline No & 247 & 92 & 37.2 & 1 & & & & & & & \\
\hline Yes & 29 & 3 & 10.3 & 0.26 & 0.11 & 0.58 & & & & & \\
\hline ICU admission & & & & & & & $<0.0001$ & & & & $<0.0001$ \\
\hline No & 175 & 41 & 23.4 & 1 & & & & 1 & & & \\
\hline Yes & 101 & 54 & 53.5 & 4.09 & 2.53 & 6.61 & & 3.42 & 2.04 & 5.75 & \\
\hline Post-acute care orientation & & & & & & & $<0.0001$ & & & & \\
\hline Post-care facilities & 65 & 38 & 58.5 & 3.59 & 2.08 & 6.19 & & & & & \\
\hline At home & 211 & 57 & 27 & 1 & & & & & & & \\
\hline Subjective functional loss at discharge & & & & & & & $<0.0001$ & & & & 0.0002 \\
\hline No & 56 & 11 & 19.6 & 1 & & & & 1 & & & \\
\hline Yes & 193 & 73 & 37.8 & 3.27 & 1.81 & 5.92 & & 3.26 & 1.75 & 6.08 & \\
\hline Hospitalisation duration (days) & & & & & & & 0.0034 & & & & \\
\hline$<7$ & 115 & 28 & 24.3 & 1 & & & & & & & \\
\hline$>7$ & 159 & 66 & 41.5 & 1.96 & 1.25 & 3.08 & & & & & \\
\hline
\end{tabular}

OR corresponding to group Discharge-/D30 - versus group Discharge+/D30-, or group Discharge+/D30- versus group Discharge+/ D30+) considering 2 OR for group Discharge-/D30 versus group Discharge+/D30+). Group Discharge-/D30-: n=125; group Discharge+/ D30-: $n=67$; group Discharge+/D30+: $n=96$.

${ }^{*}$ Only factors with a significant association at 0.1 in the bivariate model were entered into the multivariate model $(n=247)$. Stepwise variable selection with significance level for entry into the model at 0.2 and with significance level for staying in the model at 0.05 was used. Thus, variables not appearing in the multivariate model do not fulfill these selection criteria.

$\mathrm{D} 30,30$ days post-discharge; ICU, intensive care unit.

or physiotherapy, and/or diet intervention or need for post-acute care facilities.

Subjective functional loss at discharge, a stay in ICU and male sex were found predictive of the persistence of malnutrition at D30. ICU stay furthermore increased the risk of weight loss, of subjective functional loss as well as increased hospitalisation duration. Given the latter, postacute care facilities should be properly adapted for these patients; however, given the current situation, facility availability was limited due to closures, downsizing or additional placement requirements in order to mitigate the spread of COVID-19. Furthermore, patients and families may be reluctant to accept placement at a post-acute care facility. ${ }^{1}$ In our cohort, only $49 \%$ of the patients having necessitated a stay in ICU were admitted in postacute care facilities following their hospital discharge for ongoing skilled nursing care and rehabilitation; as for the remaining $51 \%$ patients, this likely represents a probable wasted opportunity. Significant loss of intrinsic capacity such as difficulties walking at a usual pace, loss of muscle mass and strength, mobility impairments, muscle weakness and poor physical performance are associated with severe adverse outcomes, increased mortality, disability, fractures and hospitalisation. ${ }^{23}$ Therefore, the early identification of any of these medical issues is important to prevent further decline and associated adverse events.

The concept of sarcopenia includes both muscle mass loss as well as muscle strength or function loss. ${ }^{10} \mathrm{SES}$ 
Table 4 Bivariate and multivariate analysis of subjective functional loss at D30 (logistic regression)

\section{Subjective \\ functional loss \\ D30}

Bivariate regression

Multivariate regression**

\begin{tabular}{|c|c|c|c|c|c|c|c|c|c|c|c|}
\hline & \multirow[b]{3}{*}{$\mathbf{N}$} & \multirow{3}{*}{$\mathrm{n}$} & \multirow[b]{3}{*}{$\%$} & \multirow[b]{3}{*}{ Odds ratio } & \multirow{2}{*}{\multicolumn{2}{|c|}{$95 \% \mathrm{Cl}^{*}$}} & \multirow{3}{*}{$P$ value } & \multirow{3}{*}{ Odds ratio } & \multirow{2}{*}{\multicolumn{2}{|c|}{$95 \% \mathrm{Cl}^{*}$}} & \multirow[b]{3}{*}{$P$ value } \\
\hline & & & & & & & & & & & \\
\hline & & & & & Lower & Upper & & & Lower & Upper & \\
\hline Age (years) & & & & & & & & 0.7656 & & & \\
\hline$<70$ & 202 & 54 & 26.7 & 1 & & & & & & & \\
\hline$>70$ & 80 & 20 & 25 & 0.91 & 0.5 & 1.66 & & & & & \\
\hline \multicolumn{4}{|c|}{ Post-acute care orientation } & & & & 0.0089 & & & & 0.0029 \\
\hline $\begin{array}{l}\text { Post-care } \\
\text { facilities }\end{array}$ & 64 & 25 & 39.1 & 2.21 & 1.22 & 4.01 & & 2.79 & 1.42 & 5.48 & \\
\hline At home & 218 & 49 & 22.5 & 1 & & & & 1 & & & \\
\hline \multicolumn{7}{|c|}{ ICU admission } & 0.0239 & & & & \\
\hline No & 183 & 40 & 21.9 & 1 & & & & & & & \\
\hline Yes & 99 & 34 & 34.3 & 1.87 & 1.09 & 3.22 & & & & & \\
\hline \multicolumn{5}{|c|}{ Subjective functional loss at discharge } & & & 0.0008 & & & & 0.0007 \\
\hline No & 57 & 1 & 1.75 & 1 & & & & 1 & & & \\
\hline Yes & 195 & 69 & 35.4 & 30.65 & 4.15 & 226.2 & & 32.63 & 4.36 & 244 & \\
\hline \multicolumn{7}{|c|}{ Malnutrition at discharge } & 0.0151 & & & & \\
\hline Absent & 116 & 23 & 19.8 & 1 & & & & & & & \\
\hline Moderate & 80 & 21 & 26.3 & 1.44 & 0.73 & 2.83 & & & & & \\
\hline Severe & 74 & 29 & 39.2 & 2.61 & 1.36 & 5.01 & & & & & \\
\hline \multicolumn{7}{|l|}{ Anosmia } & 0.0567 & & & & \\
\hline No & 160 & 49 & 30.6 & 1.71 & 0.98 & 2.98 & & & & & \\
\hline Yes & 122 & 25 & 20.5 & 1 & & & & & & & \\
\hline
\end{tabular}

D30, 30 days post-discharge; ICU, intensive care unit.

allowed assessing only functional loss perceived by the patient.

In order to describe muscle functional loss, the term 'dynapenia' has been proposed by Clark and Manini ${ }^{24}$ although this term was not retained in the EWGSOP criteria. The loss of functional capacity analysed via the SES by the patients is subjective. We hence proposed the term 'subjective functional loss' as best suited for our assessment. This tool could represent a valuable addition to the available tools currently used for the first step in screening for sarcopenia prior to confirmation of sarcopenia by mass measurement. This test could furthermore allow examining not only muscle function but also the general state of 'fatigability', whether central or peripheral.

Sarcopenia and malnutrition are two highly intertwined concepts. Muscle failure induces a reduction in chewing ability and has an impact on the foods people choose to eat, resulting in a potentially deleterious effect on dietary quality and a reduction in food diversity. Sarcopenia is also a component and severity factor of malnutrition and has recently been proposed as part of the definition of malnutrition. ${ }^{25}$ Of interest, $43.8 \%$ of our patients reported a moderate or high activity level on the IPAQ questionnaire prior to COVID-19, which did not influence muscle strength or its evolution.

In effect, the risk of sarcopenia appears extremely high on the long term after severe acute respiratory distress syndrome in other viral infections. These patients have been shown to manifest persistent functional limitation 1 year after being discharged from the ICU, largely due to muscle wasting, weakness and fatigue, involving extrapulmonary disease with impaired neuromuscular function as an important determinant of exercise limitation. ${ }^{26}$ Protein turnover is also increased in critical illness in the early days of COVID-19, as a response to massive proteolytic stimuli. Suggested mechanisms include direct muscle invasion by equine coronavirus $(\mathrm{ECoV})$ particles and immune-mediated muscle injury, although satisfactory proof of the direct invasion of SARS-CoV-2 into muscle cells is still lacking. In one postmortem skeletal muscle study based on eight SARS cases, the diagnosis of critical illness myopathy was confirmed based on clinical features. Lastly, muscle deconditioning due to immobility has been associated with diffuse atrophy at muscle biopsy. ${ }^{27}$

Some bias should be noted. In our study, the decrease in muscle strength assessed by the patients themselves 
Table 5 Bivariate and multivariate analysis of criteria combining malnutrition, subjective functional loss $(\mathrm{SES}<7 / 10)$ and performance status (PS) $>2$ (logistic regression)

\begin{tabular}{|c|c|c|c|c|c|c|c|c|c|c|c|}
\hline & \multirow[b]{3}{*}{$\mathbf{N}$} & \multicolumn{2}{|l|}{ PS } & \multicolumn{4}{|c|}{ Bivariate regression } & \multicolumn{4}{|c|}{ Multivariate regression ${ }^{*}$} \\
\hline & & \multirow[b]{2}{*}{$\mathbf{n}$} & \multirow[b]{2}{*}{$\%$} & \multirow[t]{2}{*}{ OR } & \multicolumn{2}{|l|}{$95 \% \mathrm{Cl}$} & \multirow[b]{2}{*}{$P$ value } & \multirow[t]{2}{*}{ OR } & \multicolumn{2}{|l|}{$95 \% \mathrm{Cl}$} & \multirow[b]{2}{*}{$P$ value } \\
\hline & & & & & Lower & Upper & & & Lower & Upper & \\
\hline Age (years) & & & & & & & 0.5542 & & & & \\
\hline$<70$ & 207 & 16 & 7.73 & 1 & & & & & & & \\
\hline$\geq 70$ & 81 & 8 & 9.88 & 1.31 & 0.54 & 3.19 & & & & & \\
\hline Sex & & & & & & & 0.0386 & & & & \\
\hline Male & 156 & 18 & 11.5 & 2.74 & 1.05 & 7.12 & & & & & \\
\hline Female & 132 & 6 & 4.55 & 1 & & & & & & & \\
\hline \multicolumn{2}{|c|}{ ICU admission } & & & & & & 0.0057 & 0.0057 & & & \\
\hline No & 186 & 9 & 4.84 & 1 & & & & 1 & & & \\
\hline Yes & 102 & 15 & 14.7 & 3.39 & 1.43 & 8.06 & & 3.39 & 1.43 & 8.06 & \\
\hline Anosmia & & & & & & & 0.0302 & & & & \\
\hline No & 165 & 19 & 11.5 & 3.07 & 1.11 & 8.47 & & & & & \\
\hline Yes & 123 & 5 & 4.07 & 1 & & & & & & & \\
\hline
\end{tabular}

Stepwise variable selection with significance level for entry into the model at 0.1 and with significance level for staying in the model at 0.05 was used.

*Only factors with a significant association at 0.1 in the bivariate model were entered into the multivariate model $(n=274)$.

†Quantitative variables have no reference level. The OR expresses the risk variation for a unit increase of the variable. multivariate composite indicator. Thus, variables not appearing in the multivariate model do not fulfil these selection criteria.

ICU, intensive care unit; SES, self-evaluation of strength.

was altered regardless of the BMI. It is interesting to note that while obesity was associated with more severe forms of COVID-19, in surviving patients, BMI before infection had no significant effect on the parameters studied, malnutrition and SES. However, since COVID-19 mortality was higher in obese subjects, less corpulent patients likely had a greater chance of being alive on D30. Furthermore, there are no commonly accepted criteria for sarcopenic obesity beyond those for sarcopenia and obesity separately.

It is also surprising to note that age had no significant effect on nutritional status. There are several reasons for this phenomenon. Hospital mortality was significant during the first wave of the epidemic, particularly affecting the elderly. However, overall mean age did not differ significantly between patients who could not be interviewed at D30 and the analysed cohort herein (59.6 416.7 vs $67.8 \pm 18.8, p=0.112$ ). Patients over the age of 70 years had little chance of being admitted to intensive care, thus the average age of intensive care patients was lower. This justifies the age variable being transformed into a dichotomous variable.

In patients critically ill with the severe Middle East Respiratory Syndrome (MERS) coronavirus disease, $84.6 \%$ of survivors were able to return to normal status, whereas the remaining survivors were $\operatorname{not}^{28}{ }^{29}$ based on assessment of the Karnofsky Performance Status (KPS) scale. Of particular note, a similar proportion was observed in our COVID-19 population 1 month after discharge. Such persistent exercise limitation and lower-than-normal health-related quality-of-life at $2^{30}$ and 5 years ${ }^{31}$ after ICU discharge have previously been observed, with a greater recovery in younger patients with most retaining the ability to live independently.

Olfactory and gustatory dysfunction are common symptoms in patients with COVID-19 and may represent early symptoms in the clinical course of infection. ${ }^{4}$ Anosmia was associated herein with lower food intake although it was not associated with persistent malnutrition at D30. While alteration of flavour perception may contribute to weight loss in other diseases, ${ }^{5}$ patients with olfactory disorders in the current study had less frequently subjective functional loss or severe disability at D30. The protective effect of anosmia has been reported in other studies showing that olfactory disorders less frequently necessitated oxygen therapy $^{32}$ and was associated with milder forms in patients who did not require hospitalisation. ${ }^{33}$ However, patients having undergone a stay in the ICU may have underreported their anosmia.

In our study, male patients had a higher risk of being undernourished and of developing serious disability at D30. COVID-19 has indeed shown a clear male-biased severity. This sex-biased pathogenesis is not yet fully understood, although likely multifactorial. ${ }^{34}$ The difference in immune system function may be an important determinant since numerous immune genes are present on the X chromosome. Steroid hormones could also potentially play a crucial role in pathogenesis whereby oestrogen may have immune-enhancing effects while testosterone may have immune-suppressive effects. Stress 
endurance could also represent a contributing factor in sex-biased pathogenesis from COVID-19.

Our study has several limitations, the most noteworthy being its observational nature. This study was furthermore not intended to evaluate the efficiency of homecare and rehabilitation centre management.

The present data nonetheless show the clinical relevance of the SES scale, even if potentially biased as psychasthenia, post-COVID-19 depression or over- or under-estimation depending on personality trait. The SES 10-point visual analogue scale had $87.4 \%$ sensitivity, $33.0 \%$ specificity, $64.2 \%$ positive predictive value and $66.1 \%$ negative predictive value for the diagnosis of malnutrition based on the GLIM criteria. Its high sensitivity could hence be appropriate for screening purposes, that is, detection of functional muscle loss that can be managed by adapted nutrition and tailored physical activity. However, the area under the ROC curve depicting the performance of a SES visual analogue scale score $<7$ for the diagnosis of malnutrition was 0.64 , indicating moderate predictive performance. Consequently, the interest of the SES as a tool for sarcopenia screening should be evaluated and compared with an adequate reference test, i.e. an objective value of muscle strength such as the hand grip. In our previous pilot study, ${ }^{14}$ SES was able to differentially distinguish patients with low or high progression of hand grip strength during hospitalisation $(4.7 \% \pm 5.6 \%$ vs $19.3 \% \pm 15.3 \%$; $\mathrm{p}=0.008)$. In addition, patients with SES $<7$ also had lower brachial circumference $(26.2 \pm 3.7 \mathrm{~cm}$ vs $29.5 \pm 3.8 \mathrm{~cm} ; \mathrm{p}=0.024)$ and lower calf circumference $(34.9 \pm 4.2 \mathrm{~cm}$ vs $30.7 \pm 3.7 \mathrm{~cm} ; \mathrm{p}=0.020)$.

In this study, sex and age had no independent effect on subjective functional loss although it is not known whether sex and/or age factors may explain the accuracy of SES. Men and women may respond differently to catabolic conditions due to their hormonal profiles; however, in most studies reporting gender, there was no significant association with sarcopenia prevalence (review in CruzJentoft $e t a l^{35}$ ). Moreover, there does not appear to be an independent effect of sex when comparing programmes assessing the effectiveness of supervised versus unsupervised balance and/or resistance training programmes on measures of muscle strength/power in healthy older adults. ${ }^{36}$ However, the effect of age and gender on the SES scale warrants further large-scale study.

Although international societies recommend early and systematic identification of sarcopenia, SES could represent a useful tool for muscle functional loss screening before conducting an objective measurement of muscle strength. Nevertheless, its limitations, feasibility and biases need to be investigated in larger studies.

Micronutrient deficiency was not taken into account in this study. Although previous studies have been heterogeneous in their methodological and statistical approach, most of the latter indicated a significant relationship between 25(OH)D and SARS-CoV-2 infection, COVID-19 composite severity and mortality. ${ }^{37}$ However, vitamin D has nonetheless a strong influence on muscle condition.
Thresholds for severity grading of malnutrition into moderate and SM are similar for both the GLIM and French criteria except for the BMI cut-off thresholds, which are lower in the French recommendations (17 vs 18.5 and 18.5 vs 20 for adults $<70$ years and 18 vs 20 and 21 vs 22 for adults $>70$ years). When applying the GLIM criteria, one patient would be reclassified in the malnourished group and two patients would be reclassified from MM to SM. This reclassification did not significantly modify the current results.

Lastly, ordinal polychotomous regression appears to be the most appropriate model to use with this type of data, yielding easily interpreted ORs and assuming a multiplicative model. Nevertheless, this model assumes that the level-to-level OR of the cumulative categories is similar. This is probably not always the case, since threshold effects are likely to occur, the latter being possibly identified by nominal polychotomous regression, an analysis somewhat more difficult to interpret with k1 OR for a dependent k-level variable.

\section{CONCLUSION:}

This study showed that the prevalence of SM and functional loss is extremely high in the severe forms of COVID-19. For clinical practice, nutritional support should be strengthened, particularly for patients necessitating a stay in ICU, presenting subjective functional loss at discharge and of male sex. For these reasons, screening for malnutrition and muscle functional loss should be initiated immediately at the onset of care, with the aim of improving nutritional status as well as maintaining muscle mass and physical performance. This cohort study further highlights the need to develop better care systems, by which to promote recovery and adaptation to new disability after acute infective illness. This adaptation of homecare healthcare systems should enable a quick recovery and reduce the risk of late complications.

\section{Author affiliations}

${ }^{1}$ Unité Transversale de Nutrition, CHRU de Nancy, Nancy, France

${ }^{2}$ Université Grenoble Alpes, Saint-Martin-d'Heres, France

${ }^{3}$ General Medicine Department, University of Lorraine, Nancy, France

${ }^{4}$ Internal Medicine and Clinical Immunology Department, CHRU de Nancy, Nancy,

France

${ }^{5}$ Endocrinology Diabetology and Nutrition Department, CHRU de Nancy, Nancy, France

${ }^{6}$ Medical Evaluation Department, Department of Clinical Research Support PARC, University of Lorraine, CHRU de Nancy, Nancy, France

Contributors DQ contributed to the conception and design of the research, analysis of the data and drafted the manuscript; $M G$ contributed to the conception and design of the research, acquisition and analysis of the data. P-LN-T contributed to the design of the research and analysis of the data; $\mathrm{OB}$ and $\mathrm{AM}$ contributed to the acquisition of the data; OZ, PDP, M-FV and RJ contributed to the conception and design of the research and acquisition of the data. All authors critically revised the manuscript, agree to be fully accountable for ensuring the integrity and accuracy of the work, and read and approved the final manuscript.

Funding This study was conducted with the help of the French Society for Clinical Nutrition and Metabolism.

Competing interests None declared. 
Patient and public involvement Patients and/or the public were not involved in the design, or conduct, or reporting or dissemination plans of this research.

Patient consent for publication Not required.

Provenance and peer review Not commissioned; externally peer reviewed.

Data availability statement Data are available upon reasonable request.

Open access This is an open access article distributed in accordance with the Creative Commons Attribution Non Commercial (CC BY-NC 4.0) license, which permits others to distribute, remix, adapt, build upon this work non-commercially, and license their derivative works on different terms, provided the original work is properly cited, appropriate credit is given, any changes made indicated, and the use is non-commercial. See: http://creativecommons.org/licenses/by-nc/4.0/.

\section{ORCID iD}

Didier Quilliot http://orcid.org/0000-0001-8143-9856

\section{REFERENCES}

1 Prescott HC, Girard TD. Recovery from severe COVID-19: Leveraging the lessons of survival from sepsis. JAMA 2020;324:739-740.

2 Nalbandian A, Sehgal K, Gupta A, et al. Post-Acute COVID-19 syndrome. Nat Med 2021;27:601-15.

3 Liu $\mathrm{H}$, Chen S, Liu M, et al. Comorbid chronic diseases are strongly correlated with disease severity among COVID-19 patients: a systematic review and meta-analysis. Aging Dis 2020;11:668-78.

4 Tong JY, Wong A, Zhu D, et al. The prevalence of olfactory and gustatory dysfunction in COVID-19 patients: a systematic review and meta-analysis. Otolaryngol Head Neck Surg 2020;163:3-11.

5 Roos DS, Oranje OJM, Freriksen AFD, et al. Flavor perception and the risk of malnutrition in patients with Parkinson's disease. J Neural Transm 2018;125:925-30.

$6 \mathrm{Li} \mathrm{T}$, Zhang Y, Gong C, et al. Prevalence of malnutrition and analysis of related factors in elderly patients with COVID-19 in Wuhan, China. Eur J Clin Nutr 2020;74:871-5.

7 Carod-Artal FJ. Neurological complications of coronavirus and COVID-19. Rev Neurol 2020;70:311-22.

8 Beaudart C, Rolland Y, Cruz-Jentoft AJ, et al. Assessment of Muscle Function and Physical Performance in Daily Clinical Practice : A position paper endorsed by the European Society for Clinical and Economic Aspects of Osteoporosis, Osteoarthritis and Musculoskeletal Diseases (ESCEO). Calcif Tissue Int 2019;105:1-14.

9 Cederholm T, Jensen GL, Correia MITD, et al. GLIM criteria for the diagnosis of malnutrition - A consensus report from the global clinical nutrition community. Clin Nutr 2019;38:1-9.

10 Cruz-Jentoft AJ, Bahat G, Bauer J, et al. Sarcopenia: revised European consensus on definition and diagnosis. Age Ageing 2019;48:16-31.

11 Krznarić Željko, Bender DV, Laviano A, et al. A simple remote nutritional screening tool and practical guidance for nutritional care in primary practice during the COVID-19 pandemic. Clin Nutr 2020;39:1983-7.

12 Woo J, Leung J, Morley JE. Validating the SARC-F: a suitable community screening tool for sarcopenia? J Am Med Dir Assoc 2014;15:630-4.

13 Bouëtté G, Esvan M, Apel K, et al. A visual analogue scale for food intake as a screening test for malnutrition in the primary care setting: prospective non-interventional study. Clin Nutr 2021;40:174-80.

14 Gerard M, Nguyen-Thi P-L, Malgras A, et al. Assessment of muscle function in severe and malnourished COVID-19 patients. International Journal of Nutrition and Food Sciences 2020;9:132-7.

15 Cheng MP, Papenburg J, Desjardins M, et al. Diagnostic testing for severe acute respiratory syndrome-related coronavirus 2: a narrative review. Ann Intern Med 2020;172:726-34.
16 Hewlett S, Hehir M, Kirwan JR. Measuring fatigue in rheumatoid arthritis: a systematic review of scales in use. Arthritis Rheum 2007;57:429-39.

17 Society AT. Recommended respiratory disease questionnaires for use with adults and children in epidemiologlcal research. American Review of Respiratory Disease 1978;118:7-35.

18 Oken MM, Creech RH, Tormey DC, et al. Toxicity and response criteria of the Eastern Cooperative Oncology Group. Am J Clin Oncol 1982;5:649-56.

19 Thibault R, Goujon N, Le Gallic E, et al. Use of 10-point analogue scales to estimate dietary intake: a prospective study in patients nutritionally at-risk. Clin Nutr 2009;28:134-40.

20 Craig CL, Marshall AL, Sjöström M, et al. International physical activity questionnaire: 12 -country reliability and validity. Med Sci Sports Exerc 2003;35:1381-95.

21 HAdS HAS. Diagnostic de la dénutrition de l'enfant et de l'adulte. Saint-Denis La Plaine 2019.

22 Thibault R, Quilliot D, Seguin P, et al. Nutritional care at hospital during the Covid-19 viral epidemic: expert opinion from the Frenchspeaking Society for clinical nutrition and metabolism (SFNCM). Nut Clin et Métab 2020;34:97-104.

23 Beaudart C, Rizzoli R, Bruyère O, et al. Sarcopenia: burden and challenges for public health. Arch Public Health 2014;72:45.

24 Clark BC, Manini TM. Sarcopenia =/=dynapenia. J Gerontol A Biol Sci Med Sci 2008;63:829-34.

25 Jensen GL, Cederholm T, Correia MITD, et al. GLIM criteria for the diagnosis of malnutrition: a consensus report from the global clinical nutrition community. JPEN J Parenter Enteral Nutr 2019;43:32-40.

26 Herridge MS, Chu LM, Matte A, et al. The recover program: disability risk groups and 1-year outcome after 7 or more days of mechanical ventilation. Am J Respir Crit Care Med 2016;194:831-44.

27 Msigwa SS, Wang Y, Li Y, et al. The neurological insights of the emerging coronaviruses. J Clin Neurosci 2020;78:1-7.

28 Karnofsky DA. Chemotherapy of neoplastic disease; agents of clinical value. N Engl J Med 1948;239:299-305.

29 Shalhoub S, Al-Hameed F, Mandourah Y, et al. Critically ill healthcare workers with the middle East respiratory syndrome (MERS): a multicenter study. PLoS One 2018;13:e0206831.

30 Cheung AM, Tansey CM, Tomlinson G, et al. Two-Year outcomes, health care use, and costs of survivors of acute respiratory distress syndrome. Am J Respir Crit Care Med 2006;174:538-44.

31 Herridge MS, Tansey CM, Matté A, et al. Functional disability 5 years after acute respiratory distress syndrome. N Engl J Med 2011;364:1293-304.

32 Vacchiano V, Riguzzi P, Volpi L, et al. Early neurological manifestations of hospitalized COVID-19 patients. Neurol Sci 2020;41:2029-31.

33 Yan CH, Faraji F, Prajapati DP, et al. Self-Reported olfactory loss associates with outpatient clinical course in COVID-19. Int Forum Allergy Rhinol 2020;10:821-31.

34 Pradhan A, Olsson P-E. Sex differences in severity and mortality from COVID-19: are males more vulnerable? Biol Sex Differ 2020;11:53.

35 Cruz-Jentoft AJ, Landi F, Schneider SM, et al. Prevalence of and interventions for sarcopenia in ageing adults: a systematic review. Report of the International sarcopenia initiative (EWGSOP and IWGS). Age Ageing 2014;43:748-59.

36 Lacroix A, Hortobágyi T, Beurskens R, et al. Effects of supervised vs. unsupervised training programs on balance and muscle strength in older adults: a systematic review and meta-analysis. Sports Med 2017;47:2341-61.

37 Kazemi A, Mohammadi V, Aghababaee SK, et al. Association of vitamin D status with SARS-CoV-2 infection or COVID-19 severity: a systematic review and meta-analysis. Adv Nutr 2021;18. 